\title{
Neue Ansätze für die Therapie neurologischer Erkrankungen
}

Bibliografie

Dol http://dx.doi.org/ 10.1055/s-0033-1346719

Drug Res 2013; 63, Suppl. 1: S1-S2 @ Georg Thieme

Verlag KG Stuttgart · New York . ISSN 0004-4172

Korrespondenzadresse Reinhard Hohlfeld

Ludwig-Maximilians-Universität München

Marchioninistraße 15

81377 München

reinhard.hohlfeld@

med.uni-muenchen.de

\section{Stefan Endres}

Ludwig-Maximilians-Universität München

Ziemssenstraße 1

80336 München

stefan.endres@

med.uni-muenchen.de
Noch vor 50 Jahren war die Neurologie ein vorwiegend deskriptives Fach mit nur wenigen Möglichkeiten für therapeutische Interventionen. Diese Situation hat sich grundlegend verändert. Heutzutage sind viele neurologische Erkrankungen gut therapierbar, und mehr noch: Die Therapie neurologischer Erkrankungen bildet einen wichtigen Schwerpunkt im gesamten Spektrum translationaler Forschung. Diese erfreuliche Entwicklung lässt sich besonders gut illustrieren am Beispiel der Multiplen Sklerose (MS), der häufigsten neurologischen Erkrankung junger Erwachsener. Während man in Ermangelung von Behandlungsmöglichkeiten noch in den 1970er und 80er Jahren MS-Patienten die Diagnose nicht selten verheimlichte oder das Wort „Multiple Sklerose“ euphemistisch zu umschreiben suchte, steht heute für die Therapie dieser Erkrankung ein ganzes Arsenal von kontrolliert geprüften, z.T. recht selektiven Immuntherapeutika zur Verfügung. Darüber hinaus befindet sich eine beachtliche Zahl weiterer Medikamente in verschiedenen Stadien der klinischen Prüfung. Die MS-Therapie steht im Zentrum des ersten Teils des Symposiums, wobei die aktuellen therapeutischen Entwicklungen im Kontext der Pathogenese erläutert werden.

Ein wesentlicher Schritt der pathogenetischen Kaskade der MS ist die Einwanderung von Immunzellen in das zentrale Nervensystem (ZNS), wobei die Immunzellen die Bluthirnschranke überwinden müssen. Diese „Diapedese“ ist der zentrale Angriffspunkt eines besonders wirksamen Therapeutikums, des therapeutischen monoklonalen Antikörpers Natalizumab (Vortrag Britta Engelhardt). Natalizumab bindet an $\alpha 4$-Integrin auf der Oberfläche von T-Lymphozyten und inhibiert deren Interaktion mit dem korrespondierenden Adhäsionsmolekül VCAM (vascular cell adhesion molecule) auf der Oberfläche von Endothelzellen. In Tiermodellen lässt sich die Einwanderung von Fluoreszenz-markierten Immunzellen in das ZNS-Gewebe in vivo mit Hilfe der Zwei-Photonenmikroskopie beobachten (Alexander Flügel). Ebenfalls in Tiermodellen kann die axonale Schädigung, neben Demyelinisierung und Gliose die wichtigste neuropathologische Komponente der MS, in vivo verfolgt werden. Diese Modelle sind äußerst wertvoll, um neuroprotektive Therapieansätze zu untersuchen (Martin Kerschensteiner).

Die Mehrzahl der für die MS verfügbaren bzw. in Entwicklung befindlichen Medikamente wirkt durch Immunmodulation bzw. Immunsuppression außerhalb des ZNS (Arndt Schottelius; Ralf Gold; Andrew Chan). Zu den bereits seit längerem zugelassenen „immunmodulatorischen Basistherapien“ zählen die verschiedenen Interferon-beta-Präparate (subkutane oder intramuskuläre Anwendung) sowie das Glatirameracetat. Vorwiegend bei unzureichender Wirksamkeit der Basistherapeutika werden Natalizumab bzw. Fingolimod eingesetzt. Fingolimod, ein Sphingosin-1Phosphat-Rezeptor-Modulator, war die erste zugelassene orale Therapie für die Behandlung der schubförmigen MS. Kürzlich wurde als weiteres orales Präparat Teriflunomid zugelassen. Noch für 2013 wird mit der Zulassung weiterer immunmodulierender Therapien gerechnet, und zwar für das oral einnehmbare Medikament Dimethylfumarat sowie den monoklonalen Antikörper Alemtuzumab. Letzterer bewirkt eine lang anhaltende Depletion von CD52-positiven Immunzellen. Bisherige Erfahrungen haben gezeigt, dass mit zunehmender Wirksamkeit der Medikamente auch das Risiko unerwünschter Wirkungen zunimmt. Hier ist besonders das erhöhte Infektionsrisiko zu beachten, z.T. mit Auftreten seltener opportunistischer Infektionen wie der progressiven multifokalen Leukoencephalopathie (PML), die unter Natalizumab mit einer mittleren Inzidenz zwischen 0,1 und 10 pro 1000 beobachtet wird. Bei mehr als der Hälfte der MS-Patienten verwandelt sich der ursprünglich schubförmig-remittierende Verlauf nach einigen Jahren in einen sekundär progredienten Verlauf. Bei dieser sekundär progredienten MS, ebenso wie bei der primär progredienten MS, sind immunmodulierende Therapien kaum oder gar nicht mehr wirksam. Lediglich eine immunsuppressive Substanz ist für die Therapie der sekundär progredienten MS zugelassen, das Zytostatikum Mitoxantron. Der Einsatz dieses Medikaments ist allerdings durch kumulative Kardiotoxizität und ein Therapie-assoziiertes Leukämierisiko limitiert. Große Hoffnungen richten sich daher auf neuroprotektive Therapienansätze, die allerdings bisher eher enttäuscht haben (Frauke Zipp).

Ein relativ neues Gebiet der Neuroimmunologie sind die autoimmun bedingten Enzephalitiden, wie z.B. Rasmussen-Enzephalitis und antiNMDAR-Enzephalitis. Bei der letzteren Erkrankung scheinen Autoantiköper gegen den N-Methyl-D-Aspartat-Rezeptor (NMDAR) pathogene Wirkung zu haben. Diese Erkrankungen werden häufig von Therapie-resistenten Epilepsien begleitet, die hohe therapeutische Anforderungen stellen (Christian Elger).

Der zweite Teil des Symposiums widmet sich aktuellen therapeutischen Entwicklungen bei häufigen anderen neurologischen Erkrankungen wie 
Schlaganfall (Matthias Endres; Martin Dichgans) und neurodegenerativen Erkrankungen einschließlich Morbus Alzheimer (Christian Haass; Richard Dodel), amyotropher Lateralsklerose (Albert Ludolph) und Morbus Parkinson (Heinz Reichmann). Zunehmendes Interesse finden hier insbesondere gemeinsame pathogene Mechanismen, die sowohl bei primär entzündlichen wie auch bei primär neurodegenerativen und ischämischen ZNS-Erkrankungen bedeutsam sind; sie könnten in Zukunft möglicherweise krankheitsübergreifende Therapieansätze ermöglichen. So spielen inflammatorische Mechanismen eine wichtige Rolle bei M. Alzheimer und Schlaganfall.

Abgerundet wird das Programm durch eine Diskussion neuer therapeutischer Perspektiven der Neuroonkologie, wo die VEGF (vascular endothelial growth factor)-Inhibitoren in der GliomTherapie und die hochdosierte Methotrexat-Therapie bei ZNSLymphomen besonderes Augenmerk finden (Ulrich Bogdahn). Insbesondere die neuen „Biologika“, wie z.B. monoklonale Antikörper und rekombinante Zytokine bzw. Zytokin-Inhibitoren, erfordern in hohem Maße die enge Zusammenarbeit von Kliniken,
Universitäten, forschender Industrie und regulatorischen Behörden. Traditionell bietet die Paul-Martini-Stiftung ein einzigartiges Forum, um Vertreterinnen und Vertreter dieser Bereiche zur Diskussion zusammenzubringen. Das Symposium soll daher besonders zum Diskurs zwischen universitärer und industrieller Forschung beitragen und weitere Impulse für innovative Therapiekonzepte geben.

Besonderer Dank gilt an erster Stelle allen Referenten, aber auch dem Vorstand der Paul-Martini-Stiftung und der Geschäftsstelle. Zusätzlich danken wir der Deutschen Akademie der Naturforscher Leopoldina, unter deren Mitwirkung und Schirmherrschaft dieses Symposium ausgerichtet wurde.

\section{Reinhard Hohlfeld}

Ludwig-Maximilians-Universität München

Stefan Endres

Ludwig-Maximilians-Universität München 